\title{
Cutoff dependence of the Casimir force within an inhomogeneous medium
}

\author{
S. A. R. Horsley ${ }^{1, *}$ and W. M. R. Simpson ${ }^{2,3}$ \\ ${ }^{1}$ Electromagnetic and Acoustic Materials Group, Department of Physics and Astronomy, University of Exeter, \\ Stocker Road, Exeter, EX4 4QL, United Kingdom \\ ${ }^{2}$ School of Physics and Astronomy, University of St Andrews, North Haugh, St Andrews, KY16 9SS, United Kingdom \\ ${ }^{3}$ Faculty of Physics, Weizmann Institute of Science, 234 Herzl St., Rehovot 76100, Israel
}

(Received 22 April 2013; published 22 July 2013)

\begin{abstract}
We consider the ground-state energy of the electromagnetic field in a piston geometry. In the idealized case, where the piston and the walls of the chamber are taken as perfect mirrors, the Casimir pressure on the piston is finite and independent of the small-scale physics of the media that compose the mirrors; the Casimir energy of the system can be regularized and is cutoff independent. Yet we find that, when the body of the piston is filled with an inhomogeneous dielectric medium, the Casimir energy is cutoff dependent, and the value of the pressure is thus inextricably dependent on the detailed behavior of the mirror and the medium at large wave vectors. This result is inconsistent with recent proposals for regularizing Casimir forces in inhomogeneous media.
\end{abstract}

DOI: 10.1103/PhysRevA.88.013833

PACS number(s): 42.50.Lc, 31.30.jh

\section{INTRODUCTION}

It is now a well-known and experimentally supported fact that two parallel uncharged mirrors at zero temperature will exert an attractive force upon each other, as Casimir predicted [1] - a force arising from the ground-state properties of the electromagnetic field. The theory has been made quantitative, applying to media described by $\epsilon(\omega)$ and $\mu(\omega)$ satisfying the Kramers-Kronig relations [2-5], and Casimir forces have been calculated for a variety of systems and geometries (see, e.g., $[6,7])$. The purpose of the following discussion is to demonstrate that there seems to be a problem within the theory of the Casimir effect for the case of inhomogeneous media, in spite of recent efforts to solve or circumvent it $[8,9]$. In a separate paper [10] we have shown that this problem is inherent within the Lifshitz theory of the Casimir effect, where dispersion and dissipation are properly included; the Casimir stress in such cases appears to be infinite, and resists regularization.

But this problem is not peculiar to Lifshitz theory. Here we show that, even when we make the most basic attempt to mimic dielectric media with nondispersive boundary conditionsconsidering only a simple energy summation of the field modes - the Casimir force is infinite in the limit where the regularization (cutoff) tends to infinite frequency. It seems apparent that we cannot obtain an expression for the zero-point force that is independent of the choice of cutoff in the energy summation. Our results are similar to previous findings that the Casimir energy in the presence of smooth curved boundaries is also dependent on the cutoff [11].

\section{THE CASIMIR PISTON}

We first briefly illustrate the principles of our main calculation in an idealized system where we know there exists a finite expression for the Casimir force, independent of the microscopic physics of the bodies involved. The chosen system is a cavity of length $L$, and cross-sectional area $A=L_{y} L_{z}$,

\footnotetext{
*s.horsley@exeter.ac.uk
}

divided by a mirror at a distance $a$ from the leftmost cavity wall (Fig. 1). Even at $T=0 \mathrm{~K}$ the movable mirror situated at $x=a$ is subject to a force due to the dependence of the field energy on $a$.

We can formally write the ground-state energy of the system as the sum of all possible contributions of $\hbar \omega / 2$,

$$
E=\frac{\hbar}{2} \sum_{m, p, q, \lambda}\left[\omega_{m, p, q, \lambda}^{L}+\mathrm{L} \rightarrow \mathrm{R}\right],
$$

where $\lambda \in\{1,2\}$ indicates the polarization, the range of summation for each of the indices runs over the allowed modes, and the notation " $+\mathrm{L} \rightarrow \mathrm{R}$ " indicates that the frequency for the right-hand cavity is to be added. The eigenfrequencies of the cavities to the left (L) and right (R) of $a$ are independent of the polarization when $m>0$,

$$
\begin{aligned}
& \omega_{m, p, q}^{L}=\pi c \sqrt{\frac{m^{2}}{a^{2}}+\frac{p^{2}}{L_{y}^{2}}+\frac{q^{2}}{L_{z}^{2}}}, \\
& \omega_{m, p, q}^{R}=\pi c \sqrt{\frac{m^{2}}{(L-a)^{2}}+\frac{p^{2}}{L_{y}^{2}}+\frac{q^{2}}{L_{z}^{2}}} ;
\end{aligned}
$$

when $m=0$, the $\lambda=1$ polarization is not an allowed mode of the cavity.

Given the behavior of (2), (1) is not a meaningful expression: The summand becomes ever larger as $m, p$, and $q$ are increased, and the sum diverges. This divergence is due to the artificial assumption that there exist mirrors that act at all frequencies. To fix this, a factor is inserted into the eigenfrequencies (2) to make the sum converge:

$$
\tilde{E}=\hbar \sum_{m, p, q}\left[\omega_{m, p, q}^{L} e^{-\xi \omega_{m, p, q}^{L} / c}+\mathrm{L} \rightarrow \mathrm{R}\right],
$$

where $\xi$ is a free parameter, and a factor of 2 comes from the symmetry of the system with respect to polarization. Although this is not correct for the $m=0$ mode, the energy of this mode is independent of $a$, and so is part of the energy which we ultimately neglect. As shown in Ref. [12] (see also [13,14]), when this regularization of the summation depends on frequency alone, then the result will be independent 


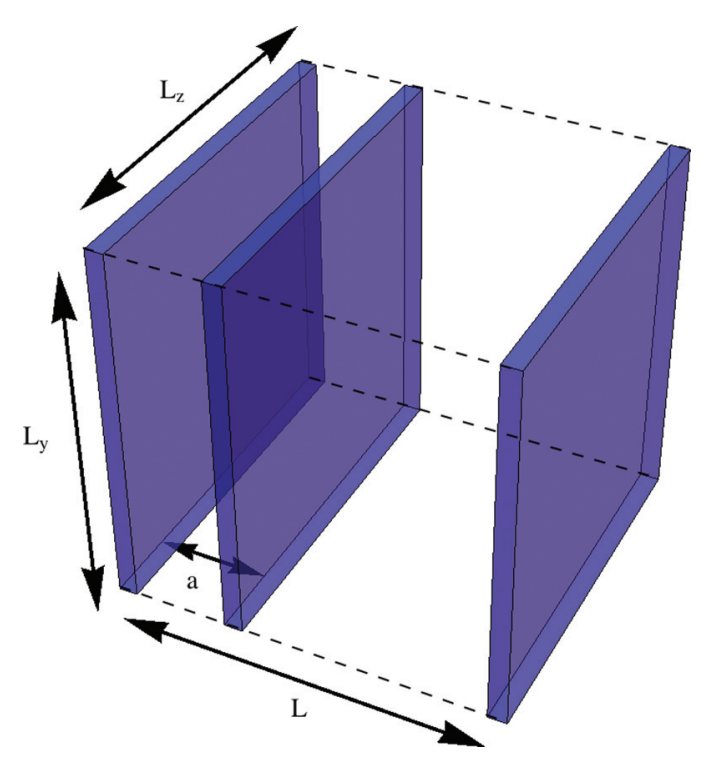

FIG. 1. (Color online) Schematic of the Casimir piston. Two fixed mirrors are positioned at $x=0$ and $x=L$, enclosed by reflecting walls at $y= \pm L_{y} / 2$ and $z= \pm L_{z} / 2$ (dashed lines). Within the chamber is vacuum, and a movable mirror at $x=a$. While the ground-state energy of the electromagnetic field in this system depends on the small-scale physics of the mirrors, the part of it depending on $a$ does not.

of the function used (in this case an exponential). This modified expression for the energy can evidently no longer be considered as the total energy of the system of field plus mirrors. Taken literally as such, (3) would imply that the eigenfrequencies of the field eventually all tend to zero, an assumption for which there is no obvious motivation. Instead, (3) should be interpreted as the part of the total energy (1) associated with the configuration of mirrors in the cavity (the energy available to do work on the mirrors). The exponential factors then amount to a model for the dispersive behavior of the mirrors: i.e., at sufficiently high frequencies and wave vectors the mirrors become transparent, and after this the total energy does not depend on their configuration.

To explicitly evaluate (3) we take the limit $L_{y} / a, L_{z} / a \rightarrow$ $\infty$, where the summation over $m$ and $p$ can be converted into an integration over $k_{y}$ and $k_{z}, \Delta k_{i}=\pi / L_{i} \rightarrow d k_{i}(i$ is either $y$ or $z$ ). After an integration over the angle, $\theta$ of $\boldsymbol{k}_{\|}=k_{y} \hat{\boldsymbol{y}}+k_{z} \hat{z}=$ $k_{\|}[\cos (\theta) \hat{\boldsymbol{y}}+\sin (\theta) \hat{\boldsymbol{z}}]$, the energy per unit area of the piston configuration is found to be

$$
\begin{aligned}
\frac{\tilde{E}}{A}= & \frac{\hbar c}{2 \pi} \sum_{m=0}^{\infty} \int_{0}^{\infty} k_{\|} d k_{\|} \sqrt{\left(\frac{m \pi}{a}\right)^{2}+k_{\|}^{2}} e^{-\xi \sqrt{(m \pi / a)^{2}+k_{\|}^{2}}} \\
& +a \rightarrow L-a,
\end{aligned}
$$

where $a \rightarrow L-a$ indicates a repetition of the previous expression with " $a$ " replaced everywhere by " $L-a$ ". To (4) we apply the identity

$$
\begin{aligned}
& \frac{d}{d k_{\|}}\left[\left(\xi^{-1} \sqrt{\left(\frac{m \pi}{a}\right)^{2}+k_{\|}^{2}}+\xi^{-2}\right) e^{-\xi \sqrt{(m \pi / a)^{2}+k_{\|}^{2}}}\right] \\
& =-k_{\|} e^{-\xi \sqrt{\left(\frac{m \pi}{a}\right)^{2}+k_{\|}^{2}}}
\end{aligned}
$$

yielding

$$
\frac{\tilde{E}}{A}=\frac{\hbar c}{\pi}\left[\frac{1}{\xi^{3}}-\frac{1}{\xi^{2}} \frac{\partial}{\partial \xi}+\frac{1}{2 \xi} \frac{\partial^{2}}{\partial \xi^{2}}\right] \sum_{m=0}^{\infty} e^{-\xi m \pi / a}+a \rightarrow L-a .
$$

The summation within (6) is a geometric series, which can be evaluated, $\sum_{m=0}^{\infty} e^{-\alpha m}=1 /\left(1-e^{-\alpha}\right)$, giving

$$
\begin{aligned}
\frac{\tilde{E}}{A}= & \frac{\hbar c}{2 \pi}\left[\frac{1}{\xi^{3}}-\frac{1}{\xi^{2}} \frac{\partial}{\partial \xi}+\frac{1}{2 \xi} \frac{\partial^{2}}{\partial \xi^{2}}\right] e^{\xi \pi / 2 a} \operatorname{cosech}(\xi \pi / 2 a) \\
& +a \rightarrow L-a
\end{aligned}
$$

The introduction of the exponential factor into (3) represents an extremely artificial model for the behavior of the mirrors at high frequencies. We therefore separate the energy into those parts that depend on $\xi$, and those that do not. In anticipation of taking the limit $\xi \rightarrow 0$, the quantity to the right of the square brackets in Eq. (7) is expanded as far as $\xi^{3}$,

$e^{\xi \pi / 2 a} \operatorname{cosech}(\xi \pi / 2 a) \sim \frac{2 a}{\xi \pi}+1+\frac{1}{3}\left(\frac{\xi \pi}{2 a}\right)-\frac{1}{45}\left(\frac{\xi \pi}{2 a}\right)^{3}$.

Inserting (8) into (7) gives finally

$$
\frac{\tilde{E}}{A}=\hbar c\left[\frac{3 L}{\pi^{2} \xi^{4}}+\frac{1}{\pi \xi^{3}}-\frac{\pi^{2}}{720 a^{3}}-\frac{\pi^{2}}{720(L-a)^{3}}\right] .
$$

As expected, the energy becomes increasingly large as $\xi \rightarrow 0$. One may interpret the $\xi^{-4}$ term as the equivalent energy in a region of free space of the same volume as the cavity. Meanwhile the $\xi^{-3}$ term comes from the $m=0$ contribution to the energy (4). However, the part of the energy depending on the position of the mirror is independent of $\xi$. One may interpret this to mean that so long as $\xi / a$ is negligibly small [i.e., neglecting the positive powers of $\xi$ in Eq. (9) is legitimate], the part of the energy that depends on $a$ is independent of how the mirror becomes transparent at high frequencies and wave vectors. Taking the derivative of (9) with respect to $a$ then yields the usual finite and $\xi$ independent pressure,

$$
\frac{F}{A}=-\frac{1}{A} \frac{\partial \tilde{E}}{\partial a}=\frac{\hbar \pi^{2} c}{240(L-a)^{4}}-\frac{\hbar \pi^{2} c}{240 a^{4}},
$$

so that the Casimir force on the mirror pulls it towards the closer of the two end walls of the chamber.

\section{THE INHOMOGENEOUS CASIMIR PISTON}

The above procedure for making (1) convergent yields terms dependent on $a$ that are all finite or zero in the limit $\xi \rightarrow 0$. Yet it is not obvious that this fortuitous situation occurs for fundamental reasons. Suppose we take the same cavity with an inhomogeneous medium within the chamber: Are the divergent terms in $\tilde{E}$ still independent of $a$ ?

Within the chamber we assume a permeability and permittivity given by (e.g., see Fig. 2)

$$
\begin{aligned}
& \mu(x)=\mu_{0}, \\
& \epsilon(x)=\epsilon_{0}[1+\delta \epsilon(x)] .
\end{aligned}
$$

It is imagined that the medium with permittivity and permeability given by (10) is a rigid body, so that we can neglect any 


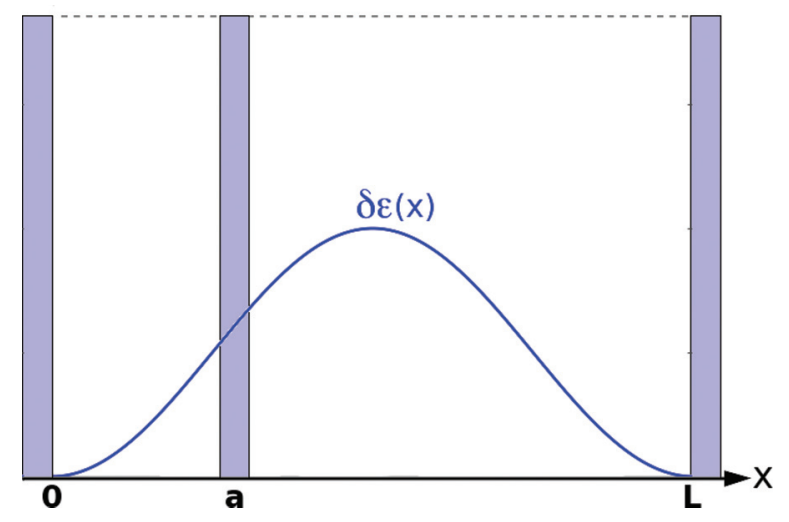

FIG. 2. (Color online) As in Fig. 1, we have a perfectly reflecting rectangular chamber of length $L$ and cross-sectional area $A$. Within the chamber is a mirror at $x=a$, surrounded by an inhomogeneous dielectric with $\epsilon$ and $\mu$ given by (10). We look for the dependence of the energy of this system on $a$.

energy associated with a deformation. We attempt to find the behavior of the force necessary to hold the mirror fixed at $a$.

As in the previous section, and in common with Casimir's original calculation [1], we make the artificial assumption that $\epsilon$ and $\mu$ are independent of frequency. This needs some justification. Dispersive effects are of course fundamental to the interaction of light and matter [15]. To properly account for them requires Lifshitz theory [2-5], and in separate work we have shown that finite answers cannot be obtained from this theory in the above case [10]. The purpose of this calculation is to examine whether this problem is peculiar to Lifshitz theory, or still present in a much more naive theory where the propagation speed of light is simply varied from point to point, and not as a function of frequency.

In general it is complicated to find the eigenfrequencies of the electromagnetic field in an inhomogeneous medium. Even with closed form expressions for these frequencies, performing the summation (3) will not be straightforward. To proceed we calculate the rate of change of the energy of the system as an inhomogeneous index profile is introduced into the cavity. The inhomogeneity is written as $\delta \epsilon(x)=\delta \alpha f(x)$, which induces a corresponding shift in the eigenfrequencies, $\delta \omega_{n, \lambda}=\delta \alpha \omega_{n, \lambda}^{(1)}$. Assuming the same regularization method as in Sec. II, the change of the energy (3) as $\delta \epsilon(x)$ is increased from zero is given by

$$
\begin{aligned}
\frac{\tilde{E}+\delta \tilde{E}}{A}= & \frac{\hbar}{4 \pi} \sum_{m, \lambda} \int_{0}^{\infty} k_{\|} d k_{\|}\left(\omega_{m, \lambda}^{L(0)}+\delta \alpha \omega_{m, \lambda}^{L(1)}\right) \\
& \times e^{-\xi\left(\omega_{m, \lambda}^{L(0)}+\delta \alpha \omega_{m, \lambda}^{L(1)}\right)}+\mathrm{L} \rightarrow \mathrm{R}
\end{aligned}
$$

the rate of change of which is

$$
\begin{aligned}
\frac{1}{A} \frac{\delta \tilde{E}}{\delta \alpha}= & \frac{\hbar}{4 \pi} \frac{\partial}{\partial \xi} \sum_{m, \lambda} \xi \int_{0}^{\infty} k_{\|} d k_{\|}\left[\omega_{m, \lambda}^{L(1)} e^{-\xi \omega_{m, \lambda}^{L(0)}\left(k_{\|}\right) / c}\right. \\
& \left.+\omega_{m, \lambda}^{R(1)} e^{-\xi \omega_{m, \lambda}^{R(0)}\left(k_{\|}\right) / c}\right] .
\end{aligned}
$$

To calculate (12) we now determine these first-order shifts in the eigenfrequencies. The situation is slightly more involved than Sec. II, due to the fact that the two polarizations do not behave degenerately in the medium defined by (10).
To calculate the change in the eigenfrequencies of the cavity we use first-order perturbation theory. Within the cavity, the electromagnetic field obeys

$$
\boldsymbol{\nabla} \times \boldsymbol{\nabla} \times \boldsymbol{E}_{m, \lambda}-\frac{\omega_{m, \lambda}^{2}}{c^{2}}[1+\delta \epsilon(x)] \boldsymbol{E}_{m, \lambda}=0,
$$

where $m$ labels the spatial dependence of the mode, and $\lambda \epsilon$ $\{1,2\}$ the polarization. When $\delta \epsilon=0$, the modes in the region $x \in[0, a]$ are given by

$$
\boldsymbol{E}_{m, 1}^{(0)}=\hat{\boldsymbol{x}} \times \hat{\boldsymbol{k}}_{\|} \sqrt{\frac{2}{a A}} \sin (m \pi x / a) e^{i \boldsymbol{k}_{\|} \cdot \boldsymbol{x}},
$$

and

$$
\begin{aligned}
\boldsymbol{E}_{m, 2}^{(0)}= & \sqrt{\frac{2 / a A}{k_{\|}^{2}+(m \pi / a)^{2}}}\left[k_{\|} \hat{\boldsymbol{x}} \cos (m \pi x / a)\right. \\
& \left.-i \hat{\boldsymbol{k}}_{\|}\left(\frac{m \pi}{a}\right) \sin (m \pi x / a)\right] e^{i \boldsymbol{k}_{\|} \cdot \boldsymbol{x}},
\end{aligned}
$$

where we assume the limit of $L_{y} / a, L_{z} / a \rightarrow \infty$ as in Sec. II. After substituting, $a \rightarrow L-a$, the modes in the region $x \in[a, L]$ are also given by (14) and (15). All modes are normalized over the volume $V$ of each region of the chamber, $\int_{V}\left|\boldsymbol{E}_{m, \lambda}^{(0)}\right|^{2} d^{3} \boldsymbol{x}=1$.

Writing the field as $\boldsymbol{E}_{m, \lambda}=\boldsymbol{E}_{m, \lambda}^{(0)}+\delta \alpha \boldsymbol{E}_{m, \lambda}^{(1)}$, we find that to first order in $\delta \alpha$,

$$
\begin{aligned}
\nabla \times & \nabla \times \boldsymbol{E}_{m, \lambda}^{(1)}-\frac{1}{c^{2}}\left[2 \omega_{m, \lambda}^{(1)} \omega_{m, \lambda}^{(0)} \boldsymbol{E}_{m, \lambda}^{(0)}\right. \\
& \left.+\omega_{m, \lambda}^{(0) 2} \boldsymbol{E}_{m, \lambda}^{(1)}+\omega_{m, \lambda}^{(0) 2} \boldsymbol{E}_{m, \lambda}^{(0)} f(x)\right]=0 .
\end{aligned}
$$

Multiplying (16) on the left by $\boldsymbol{E}_{m, \lambda}^{(0) \star}$ and integrating over $V$ (which could either be the left or right region of Fig. 2), after an integration by parts we find

$$
\omega_{m, \lambda}^{(1)}=-\frac{1}{2} \omega_{m, \lambda}^{(0)} \int_{V}\left|\boldsymbol{E}_{m, \lambda}^{(0)}\right|^{2} f(x) d^{3} \boldsymbol{x},
$$

which is the standard expression for the first-order perturbation of the eigenfrequencies of an optical cavity (see, e.g., $[16,17]$ ). We consider the particular case illustrated in Fig. 2:

$$
f(x)=\frac{1}{2}[1-\cos (2 \pi x / L)] .
$$

We emphasize that we are considering some arbitrary fixed value of $L$. The quantity $L$ only occurs in this expression in order to make the permittivity equal to that of free space at the edges of the cavity. We find after inserting (14) and (15) into (17), that in the leftmost portion of the piston the first-order change in the frequencies is given by

$$
\omega_{m, 1}^{L(1)}=-\frac{1}{4} \omega_{m, 1}^{L(0)}\left[1+\frac{\sin (2 \pi a / L)}{2 \pi} \frac{L m^{2} / a}{(a / L)^{2}-m^{2}}\right],
$$

except when $m=0$, in which case $\omega_{m, 1}^{L(1)}=0$. The eigenfrequency of the second polarization gets shifted by

$$
\begin{aligned}
\omega_{m, 2}^{L(1)}= & -\frac{1}{4} \omega_{m, 2}^{L(0)}\left\{1+\delta_{m 0}-\frac{\sin (2 \pi a / L)}{2 \pi}\right. \\
& \left.\times\left[\frac{L}{a}+\left(\frac{k_{\|}^{2}-\left(\frac{m \pi}{a}\right)^{2}}{k_{\|}^{2}+\left(\frac{m \pi}{a}\right)^{2}}\right) \frac{a / L}{(a / L)^{2}-m^{2}}\right]\right\} .
\end{aligned}
$$


After substituting $a \rightarrow L-a$ into (19) and (20), one has the expressions for the right-hand side of the cavity. The rate of change in energy per unit area due to the inhomogeneity of the medium is computed through inserting (19) and (20) into (12). The integration over $k_{\|}$is performed using the same techniques as in Sec. II. The results are given by (A2) and (A3) in the Appendix. In addition to terms proportional to geometric series [cf. (6)], the resulting sum over $m$ contains terms of the form

$$
\Phi\left(e^{-\xi \pi / a}, 1, v\right)=\sum_{m=0}^{\infty} \frac{e^{-\xi m \pi / a}}{m+v} .
$$

The quantity $\Phi$ is known as the Lerch function [18,19]. Applying the notation of (21), we can perform the summation, with the result

$$
\begin{aligned}
\frac{1}{A} \frac{\delta \tilde{E}}{\delta \alpha}= & -\frac{\hbar c}{16 \pi} \frac{\partial}{\partial \xi} \xi\left\{\left(1-\frac{\sin (2 \pi a / L)}{2 \pi a / L}\right)\left(\frac{2}{\xi^{3}}-\frac{2}{\xi^{2}} \frac{\partial}{\partial \xi}+\frac{1}{\xi} \frac{\partial^{2}}{\partial \xi^{2}}\right) e^{\xi \pi / 2 a} \operatorname{cosech}(\xi \pi / 2 a)\right. \\
& \left.-\frac{\sin (2 \pi a / L)}{2 \pi} \frac{1}{\xi} \frac{\partial^{2}}{\partial \xi^{2}}\left[\Phi\left(e^{-\xi \pi / a}, 1, a / L\right)-\Phi\left(e^{-\xi \pi / a}, 1,-a / L\right)\right]\right\}+a \rightarrow L-a .
\end{aligned}
$$

Equation (22) represents the rate of change of $\tilde{E}$ as the amplitude of $\delta \epsilon$ is increased from zero to $\delta \alpha$. Again we consider the limit $\xi \rightarrow 0$, and make use of the following series expansion of the Lerch function [20]:

$$
\begin{aligned}
\Phi\left(e^{-\xi \pi / a}, 1, v\right)= & -e^{\xi \pi v / a}[\gamma+\psi(v)+\ln (\xi \pi / a) \\
& \left.+\sum_{m=0}^{\infty}(-1)^{m+1} \frac{B_{m+1}(v)}{m+1} \frac{(\pi \xi / a)^{m+1}}{(m+1) !}\right],
\end{aligned}
$$

where $\gamma$ is Euler's constant, $\psi(v)$ is the digamma function (logarithmic derivative of the gamma function), and the $B_{m+1}(v)$ are Bernoulli polynomials [18]. In the regime of interest-where $\xi \ll 1$ and $0<v<1$ - the expansion (23) is well behaved. From (23) we find, after neglecting positive powers of $\xi$,

$$
\begin{aligned}
\frac{1}{\xi} \frac{\partial^{2}}{\partial \xi^{2}}\left[\Phi\left(e^{-\xi \pi / a}, 1, v\right)-\Phi\left(e^{-\xi \pi / a}, 1,-v\right)\right] \\
\sim-\frac{2 \pi v}{a \xi^{2}}-2\left(\frac{\pi v}{a}\right)^{3} \ln (\xi \pi / a) \\
\quad-\frac{\pi^{2} v}{a^{2} \xi}\{1+v[\psi(v)-\psi(-v)]\} \\
-\left(\frac{\pi}{a}\right)^{3} v\left\{v^{2}[2(\gamma-1)+\psi(v)+\psi(-v)]+1 / 6\right\} .
\end{aligned}
$$

Applying (24) and (8) to (22) gives the following expression for the rate of change of energy with respect to $\alpha$ :

$$
\begin{aligned}
\frac{1}{A} \frac{\delta \tilde{E}}{\delta \alpha}= & \frac{\hbar c}{4 \pi}\left[\left(1-\frac{\sin (2 \pi a / L)}{2 \pi a / L}\right)\left(\frac{9 a}{\xi^{4} \pi}+\frac{1}{\xi^{3}}+\frac{\pi^{3}}{720 a^{3}}\right)\right. \\
& +\frac{\sin (2 \pi a / L)}{4 \pi}\left\{\frac{\pi}{L \xi^{2}}-\frac{\pi^{3}}{L^{3}}[\ln (\xi \pi / a)+1]\right. \\
& +\frac{\pi^{3}}{2 L^{3}}[2(\gamma-1)+\psi(a / L)+\psi(-a / L)] \\
& \left.\left.+\frac{\pi^{3}}{12 a^{2} L}\right\}\right]+a \rightarrow L-a .
\end{aligned}
$$

Evidently the divergent terms are, in general, dependent on the position of the piston within the chamber [21]. These divergent terms are not canceled by including the contributions to the energy from the other side of the piston, as they were in Eq. (9). This means that in the limit $\xi \rightarrow 0$, the force on the piston is discontinuous as a function of $\alpha$, being finite when $\alpha=0$, and infinite as $\alpha$ is moved away from zero. There is one exception, and that is when the mirror is at the center of the cavity ( $a=$ $L / 2$ ). Then the rate of change of the energy is

$$
\frac{1}{A} \frac{\delta \tilde{E}}{\delta \alpha}=\frac{\hbar c}{4 \pi}\left(\frac{9 L}{\xi^{4} \pi}+\frac{1}{\xi^{3}}+\frac{\pi^{3}}{720 a^{3}}+\frac{\pi^{3}}{720(L-a)^{3}}\right),
$$

which yields a cutoff independent force. This is consistent with our previous finding that cutoff independent results can only be obtained when one considers regions of space where the local gradient of both $\epsilon$ and $\mu$ is zero [10]. For the profile given in Eq. (18), both the mirror and the cavity walls are within such regions when $a=L / 2$. In general, however, it seems that a $\xi$ independent meaning cannot be given to the Casimir force when in an inhomogeneous medium.

\section{RELATION TO PREVIOUS PROPOSALS}

It was recently proposed [9] that one should calculate the Casimir force in an inhomogeneous medium through forming a Laurent expansion of the energy (or pressure) in powers of the regularizing parameter (here $\xi$ ). The regularized quantity is then defined to be the term $c_{0}$ in the Laurent expansion that is independent of the regularizing parameter. This procedure works well for homogeneous systems. However, applying it to (25) (which is proportional to the change in energy due to the inhomogeneous medium, when multiplied by $\delta \alpha$ ) proves problematic. For one thing, we would still be left with the logarithmic divergence. More seriously, however, the negative powers of $\xi$ which depend on $a$ are simply swept away. Therefore the regularization procedure modifies the value of the force on the mirrors. There is no obvious physical justification for this and we emphasize, as we did in Sec. II, that the regularization in the case of a homogeneous medium does not modify the value of the force (the diverging terms are independent of the positions of the mirrors). With our method of imposing a cutoff (which is equivalent to the usual 
technique of introducing an exponential within the summation over frequencies [6]) we find that the force depends on the scale of the cutoff.

\section{CONCLUSIONS}

It has been demonstrated in previous work [10] that there is a problem with the Lifshitz theory of Casimir forces: The Casimir stress in inhomogeneous media, where the optical properties of the medium vary continuously in space, is infinite and resists regularization. But the problem of computing Casimir forces in inhomogeneous media appears to be systemic. Here we have shown that, even with a simple energy mode summation, the ground-state energy of a system proves similarly resistive to regularization. We have shown this for the case of a Casimir piston, when an inhomogeneous medium is present in the cavity. Our calculation demonstrates a cutoff dependence in the Casimir force which suggests, surprisingly, that the Casimir forces in a system might depend in detail on its microphysical properties. If this is the case, it seems unlikely that a generally finite and physically meaningful result could be obtained through a simple modification to the existing regularization procedure $[9,22,23]$.

\section{ACKNOWLEDGMENTS}

The authors wish to thank T. G. Philbin, U. Leonhardt, and Y. Imry for useful discussions, as well as the referee for suggesting the index profile (18), which proved more illuminating than the original expression. S.A.R.H. thanks the EPSRC for financial support, and the Weizmann Institute for their hospitality. W.M.R.S. thanks SUPA and the Weizmann Institute for their financial support.

\section{APPENDIX: INTEGRATION OF PERTURBED EIGENFREQUENCIES}

Here we give the results of the following integrals:

$$
I_{m, \lambda}^{s}=\int_{0}^{\infty} k_{\|} d k_{\|} \omega_{m, \lambda}^{s(1)} e^{-\xi \omega_{m, \lambda}^{s(0)}\left(k_{\|}\right) / c},
$$

where $s \in\{L, R\}$ the $\omega_{m, \lambda}^{s(1)}$ are given by (19) and (20), with the $s=R$ expressions obtained after the substitution $a \rightarrow L-a$. The eigenfrequency shifts for polarization, $\lambda=1$ depends on $k_{\|}$only through $\omega_{m, 1}^{s(0)}$ : i.e., in the same way as the unperturbed eigenfrequencies. Therefore (A1) can be performed for this polarization in the same way as Sec. II, with the result

$$
\begin{aligned}
I_{m, 1}^{L}= & -\frac{c}{4}\left(1-\delta_{m 0}\right)\left[1+\frac{\sin (2 \pi a / L)}{2 \pi} \frac{L m^{2} / a}{(a / L)^{2}-m^{2}}\right] \\
& \times\left(\frac{2}{\xi^{3}}-\frac{2}{\xi^{2}} \frac{\partial}{\partial \xi}+\frac{1}{\xi} \frac{\partial^{2}}{\partial \xi^{2}}\right) e^{-\xi m \pi / a} .
\end{aligned}
$$

Polarization $\lambda=2$ has a slightly more complicated dependence on $k_{\|}$. Yet there is nothing fundamentally different about performing the integrals, and one obtains

$$
\begin{aligned}
I_{m, 2}^{L}= & -\frac{c}{4}\left\{\left[1+\delta_{m 0}-\frac{\sin (2 \pi a / L)}{2 \pi}\left(\frac{L}{a}+\frac{a / L}{(a / L)^{2}-m^{2}}\right)\right]\right. \\
& \times\left(\frac{2}{\xi^{3}}-\frac{2}{\xi^{2}} \frac{\partial}{\partial \xi}+\frac{1}{\xi} \frac{\partial^{2}}{\partial \xi^{2}}\right) \\
& \left.+\frac{\sin (2 \pi a / L)}{2 \pi}\left(\frac{2 a / L}{(a / L)^{2}-m^{2}}\right) \frac{1}{\xi} \frac{\partial^{2}}{\partial \xi^{2}}\right\} e^{-\xi m \pi / a} .
\end{aligned}
$$

After substituting $a \rightarrow L-a$ into (A2) and (A3), one obtains $I_{m, 1}^{R}$ and $I_{m, 2}^{R}$.
[1] H. B. G. Casimir, Koninkl. Ned. Akad. Wetenschap. 51, 793 (1948).

[2] E. M. Lifshitz, Zh. Eksp. Teor. Fiz. 29, 94 (1955).

[3] I. E. Dzyaloshinskii, E. M. Lifshitz, and L. P. Pitaevskii, Adv. Phys. 10, 165 (1961).

[4] E. M. Lifshitz and L. P. Pitaevskii, Statistical Physics (Part 2) (Butterworth-Heinemann, Oxford, UK, 2003).

[5] T. G. Philbin, New J. Phys. 13, 063026 (2011).

[6] M. Bordag, G. L. Klimchitskaya, U. Mohideen, and V. M. Mostepanenko, Advances in the Casimir Effect (Oxford University Press, New York, 2009).

[7] D. Dalvit, P. Milonni, D. Roberts, and F. de Rosa, Casimir Physics (Springer-Verlag, Berlin, 2011).

[8] F. S. S. Rosa, D. A. R. Dalvit, and P. W. Milonni, Phys. Rev. A 84, 053813 (2011).

[9] S. I. Goto, A. C. Hale, R. W. Tucker, and T. J. Walton, Phys. Rev. A 85, 034103 (2012).

[10] W. M. R. Simpson, S. A. R. Horsley, and U. Leonhardt, Phys. Rev. A 87, 043806 (2013).

[11] E. B. Kolomeisky, J. P. Straley, L. S. Langsjoen, and H. Zaidi, J. Phys. A 43, 385402 (2010).

[12] G. Plunien, B. Müller, and W. Greiner, Phys. Rep. 134, 87 (1986).

[13] C. R. Hagen, Eur. Phys. J. C 19, 677 (2001).
[14] C. R. Hagen, arXiv:quant-ph/0102135.

[15] L. D. Landau, E. M. Lifshitz, and L. P. Pitaevskii, The Electrodynamics of Continuous Media (Butterworth-Heinemann, Oxford, 2004).

[16] D. Pozar, Microwave Engineering (Wiley, New York, 1998).

[17] S. G. Johnson, M. Ibanescu, M. A. Skorobogatiy, O. Weisberg, J. D. Joannopoulos, and Y. Fink, Phys. Rev. E 65, 066611 (2002).

[18] H. Bateman, Higher Transcendental Functions (McGraw-Hill, New York, 1953), Vol. 1.

[19] I. S. Gradshteyn and I. M. Ryzhik, Table of Integrals, Series, and Products (Elsevier, New York, 2007).

[20] An examination of [19] makes it appear as though the expansion (23) does not apply to the case when the middle index equals unity. However [18] shows that it does hold in this case, it is only that the expansion can be simplified to a hypergeometric function (we do not require this simplification). We have also verified (23) by direct numerical evaluation, comparing it to the results of (21).

[21] We have numerically checked that (25) agrees with (12) for small $\xi$.

[22] T. G. Philbin, C. Xiong, and U. Leonhardt, Ann. Phys. 325, 579 (2009).

[23] U. Leonhardt and W. M. R. Simpson, Phys. Rev. D 84, 081701 (2011). 